\title{
First muon acceleration using a radio-frequency accelerator
}

\author{
S. Bae,${ }^{1,2}$ H. Choi,${ }^{1,2}$ S. Choi, ${ }^{1,2}$ Y. Fukao,${ }^{3}$ K. Futatsukawa, ${ }^{3}$ K. Hasegawa, ${ }^{4}$ T. Iijima, ${ }^{5}$ \\ H. Iinuma, ${ }^{6}$ K. Ishida, ${ }^{7}$ N. Kawamura, ${ }^{3}$ B. Kim, ${ }^{1,2}$ R. Kitamura, ${ }^{8}$ H. S. Ko, ${ }^{1,2}$ Y. Kondo, ${ }^{4}{ }^{*}$ S. Li ${ }^{8}$ \\ T. Mibe, ${ }^{3}$ Y. Miyake, ${ }^{3}$ T. Morishita, ${ }^{4}$ Y. Nakazawa, ${ }^{6}$ M. Otani, ${ }^{3, \dagger}$ G. P. Razuvaev, ${ }^{9,10,11}$ N. Saito, ${ }^{12}$ \\ K. Shimomura, ${ }^{3}$ Y. Sue, ${ }^{5}$ E. Won, ${ }^{13}$ and T. Yamazaki ${ }^{3}$ \\ ${ }^{1}$ Seoul National University, Seoul 08826, Republic of Korea \\ ${ }^{2}$ Institute for Nuclear and Particle Astrophysics, Seoul National University, \\ Seoul, 08826, Republic of Korea \\ ${ }^{3}$ High Energy Accelerator Research Organization (KEK), Tsukuba, Ibaraki 305-0801, Japan \\ ${ }^{4}$ Japan Atomic Energy Agency (JAEA), Tokai, Naka, Ibaraki 319-1195, Japan \\ ${ }^{5}$ Nagoya University, Nagoya, Aichi 464-8602, Japan \\ ${ }^{6}$ Ibaraki University, Mito, Ibaraki 310-8512, Japan \\ ${ }^{7}$ Riken, Wako, Saitama 351-0198, Japan \\ ${ }^{8}$ University of Tokyo, Hongo, Tokyo 171-8501, Japan \\ ${ }^{9}$ Budker Institute of Nuclear Physics, SB RAS, Novosibirsk 630090, Russia \\ ${ }^{10}$ Novosibirsk State University, Novosibirsk 630090, Russia \\ ${ }^{11}$ Pulkovo Observatory, St. Petersburg, 196140, Russia \\ ${ }^{12}$ J-PARC Center, Tokai, Naka, Ibaraki 319-1195, Japan \\ ${ }^{13}$ Korea University, Seoul 02841, Republic of Korea
}

(Received 2 February 2018; published 18 May 2018)

\begin{abstract}
Muons have been accelerated by using a radio-frequency accelerator for the first time. Negative muonium atoms $\left(\mathrm{Mu}^{-}\right)$, which are bound states of positive muons $\left(\mu^{+}\right)$and two electrons, are generated from $\mu^{+}$'s through the electron capture process in an aluminum degrader. The generated $\mathrm{Mu}^{-}$'s are initially electrostatically accelerated and injected into a radio-frequency quadrupole linac (RFQ). In the RFQ, the $\mathrm{Mu}^{-}$'s are accelerated to $89 \mathrm{keV}$. The accelerated $\mathrm{Mu}^{-}$'s are identified by momentum measurement and time of flight. This compact muon linac opens the door to various muon accelerator applications including particle physics measurements and the construction of a transmission muon microscope.
\end{abstract}

DOI: $10.1103 /$ PhysRevAccelBeams.21.050101

\section{INTRODUCTION}

Since its invention, the radio-frequency (rf) accelerator has accelerated a wide variety of particles from electrons to rare isotopes, and greatly contributed to the progress of various branches of science. Recently, the demand for muon acceleration has arisen not only in the field of elementary particle physics, but also in material and life sciences. For example, in muon collider and neutrino factory studies [1], it is proposed that the large transverse emittance of the muon beam can be reduced using ionization cooling [2]. A muon beam passes through a material, and subsequently the lost energy in the material is restored using rf acceleration. After all the cooling processes, muons

\footnotetext{
yasuhiro.kondo@j-parc.jp

masashio@post.kek.jp
}

Published by the American Physical Society under the terms of the Creative Commons Attribution 4.0 International license. Further distribution of this work must maintain attribution to the author(s) and the published article's title, journal citation, and DOI. are accelerated from a few $\mathrm{MeV}$ with rf accelerators. In material and life sciences, one promising application of muon acceleration is in the construction of a transmission muon microscope. If the muons can be cooled to the thermal temperature [ultraslow muon (USM)] and subsequently reaccelerated, transmission muon microscopes will be realized [3]. The remarkable progress made with modern proton drivers enables the USM generator to be used as a particle source of accelerators. Because the mass of the muon is 200 times larger than that of the electron, the transmission depth of a $10 \mathrm{MeV}$ muon reaches approximately $10 \mu \mathrm{m}$. This enables three-dimensional imaging of living cells, which is impossible with the use of transmission electron microscopes. Another application of USM acceleration is precise measurement of the muon anomalous magnetic moment $a_{\mu}=(g-2)_{\mu} / 2$ and electric dipole moment (EDM). Muon acceleration is essential to realize these applications; however, it has not been demonstrated except for simple electrostatic acceleration. In this paper, the first demonstration of muon rf acceleration is presented. It was conducted during the development of the 
muon rf linear accelerator (linac) for a muon $g-2 / \mathrm{EDM}$ experiment.

\section{A $g-2$ /EDM EXPERIMENT AT J-PARC}

The $(g-2)_{\mu}$ anomaly is one of the most promising methods to explore physics beyond the Standard Model of elementary particle physics. Currently, the most precise measurement of $a_{\mu}$ has been performed by the E821 experiment of Brookhaven National Laboratory [4]. The precision is $0.54 \mathrm{ppm}$, and the measured value is approximately 3 standard deviations from the Standard Model prediction [5-7]. To improve this precision, a new muon $g-2 /$ EDM experiment is proposed at Japan Proton Accelerator Research Complex (J-PARC). This experiment, J-PARC E34, aims to measure $a_{\mu}$ with a precision of $0.1 \mathrm{ppm}$ and the EDM with a precision of $1 \times 10^{-21} e \cdot \mathrm{cm}$ [8]. Unlike E821 and its predecessors and successor [9], E34 will use a low-emittance muon beam. The required transverse momentum spread $\Delta p_{t} / p$ is less than $10^{-5}$, and the assumed transverse emittance is $1.5 \pi \mathrm{mm}$ mrad. To satisfy this requirement, the $25 \mathrm{meV}$ USMs generated by laser dissociation of the thermal muoniums ( $\mathrm{Mu}$, or $\mu^{+} e^{-}$) from a silica aerogel target [10] will be accelerated to $212 \mathrm{MeV}$ using a muon linac [11]. The muon linac will be constructed at the $\mathrm{H}$ line [12] of the J-PARC muon science facility (MUSE) [13]. It will consist of a radio-frequency quadrupole linac (RFQ) [14], an interdigital H-mode drift tube linac [15], disk-and-washer coupled cell linac [16], and disk loaded traveling wave structures [17].

The muon linac is an unproven technology, and thus muon acceleration should be demonstrated as soon as possible prior to the construction of the actual linac. In addition, because the intensity of the muon linac is much lower than that of an ordinary linac, a commissioning method should be established through a muon acceleration experiment. The laser-dissociation USM source is now being developed with high priority; however, an earlier and simpler slow muon source is necessary to conduct the muon acceleration experiment. The scheme of muon cooling using a simple metal degrader and reacceleration with an RFQ, originally proposed at Los Alamos National
Laboratory [18], is suitable for this purpose. We basically follow this method, but the emittance of the simply degraded muon is too large compared to the RFQ acceptance. Therefore, we use epithermal negative muoniums $\left(\mathrm{Mu}^{-}\right.$, or $\left.\mu^{+} e^{-} e^{-}\right)$generated from degraded $\mu^{+}$'s through the electron capture process [19]. The emittance of this $\mathrm{Mu}^{-}$beam is still larger than the RFQ acceptance, but much smaller than that of the degraded $\mu^{+}$'s. Moreover, the energy spectrum of simply degraded $\mu^{+}$'s is very broad, reaching up to the full RFQ accelerated energy. Some of those penetrate the RFQ without acceleration. We call these $\mu^{+}$'s penetrating $\mu^{+}$'s. This makes it difficult to distinguish them from the accelerated $\mu^{+}$'s. In contrast, the $\mathrm{Mu}^{-}$'s have a sharp peak near zero energy, and the accelerated $\mathrm{Mu}^{-}$'s are easily separated from the penetrating $\mu^{+}$'s because they have opposite charge. Using this slow $\mathrm{Mu}^{-}$source and a prototype RFQ of the J-PARC linac [20], we conducted the muon acceleration experiment in a multipurpose experimental area (D2 area) of MUSE.

\section{EXPERIMENTAL APPARATUS}

Figure 1 shows a schematic drawing of the experimental setup. The MUSE facility provides a pulsed surface muon $\left(\mu^{+}\right)$beam produced by $\pi^{+}$decay near the surface of the production target. The beam pulse width is $47 \mathrm{~ns}$ in rms, and the repetition rate is $25 \mathrm{~Hz}$ [21]. For this experiment, the beam power of the J-PARC Rapid Cycling Synchrotron (RCS) was $300 \mathrm{~kW}$. The energy of the $\mu^{+}$beam was chosen to be $2.9 \mathrm{MeV}$ to maximize the $\mathrm{Mu}^{-}$emission yield on the basis of a separate experiment [22], which was conducted prior to this acceleration experiment, and individually measured $\mu^{+}$deceleration and $\mathrm{Mu}^{-}$production itself. With this beam line setting, the $\mu^{+}$intensity was estimated to be $3 \times 10^{6} / \mathrm{s}$. The $\mu^{+}$'s were incident on an aluminum degrader with dimensions $43 \times 40 \mathrm{~mm}^{2}$ and a thickness of $200 \mu \mathrm{m}$. The beam profile of the $\mu^{+}$at the Al degrader was estimated from a beam line simulation based on the G4BEAMLINE simulation package [23]. The estimated profile was verified by comparison with the measured profile at the $\mu^{+}$focal point of the D2 area. The position of the $\mathrm{Al}$ degrader is different from the $\mu^{+}$focal point due to a

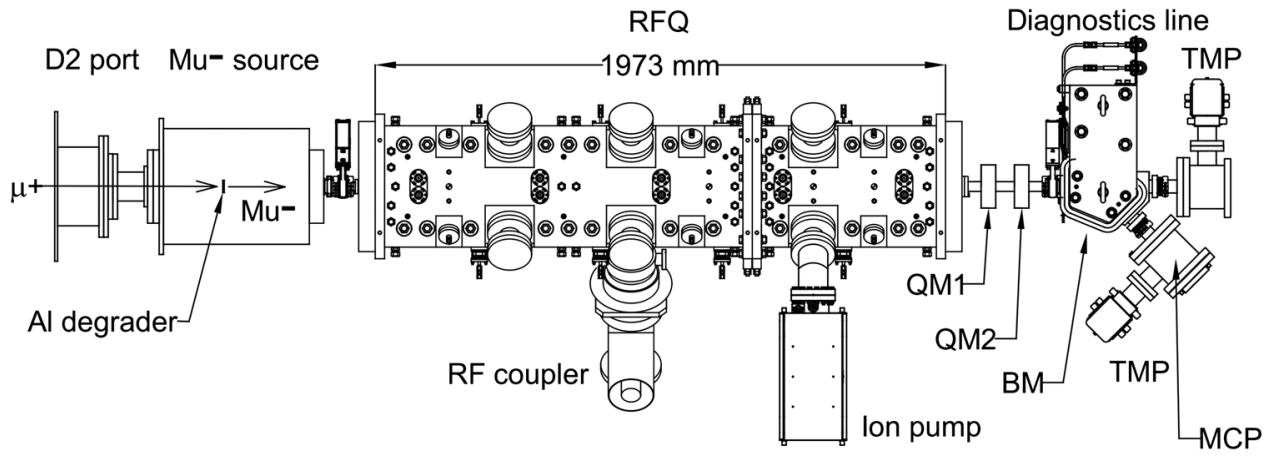

FIG. 1. Schematic drawing of the experimental setup. 
TABLE I. RFQ parameter conversion from $\mathrm{H}^{-}$to $\mu$.

\begin{tabular}{llll}
\hline \hline Particle & $\mathrm{H}^{-}$ & & $\mu$ \\
\hline Mass $\left(\mathrm{MeV} / c^{2}\right)$ & 939.3 & & 105.7 \\
Injection $\beta$ & & 0.010 & \\
Injection energy $(\mathrm{keV})$ & 50 & & 5.6 \\
Extraction $\beta$ & & 0.041 & \\
Extraction energy $(\mathrm{keV})$ & 810 & & 89 \\
Intervane voltage $(\mathrm{kV})$ & 80.7 & & 9.08 \\
Nominal power $(\mathrm{kW})$ & 180 & & 2.3 \\
\hline \hline
\end{tabular}

geometrical constraint, and therefore the horizontal and vertical rms beam sizes at the $\mathrm{Al}$ degrader were estimated from the simulation to be 32 and $28 \mathrm{~mm}$, respectively; thus, only $27 \%$ of the primary $\mu^{+}$hit the $\mathrm{Al}$ degrader. The rms energy spread is estimated to be $16 \%$. The $\mu^{+}$'s were decelerated through the $\mathrm{Al}$ degrader, and some $\mu^{+}$'s captured two electrons to become $\mathrm{Mu}^{-}$'s at the downstream surface of the $\mathrm{Al}$ degrader. The conversion efficiency from $\mu^{+}$to $\mathrm{Mu}^{-}$is estimated to be $8 \times 10^{-7}$ from the data of the separate experiment [22]. By using an electrostatic lens system, a Soa lens [24], the generated $\mathrm{Mu}^{-}$'s were accelerated to $5.6 \mathrm{keV}$ and focused on the entrance of the RFQ.

As mentioned above, the prototype RFQ of the J-PARC linac was used for this experiment. The length of this RFQ corresponds to two-thirds of the length of the complete $3 \mathrm{MeV}$ RFQ, and this prototype RFQ was designed to accelerate negative hydrogen ions $\left(\mathrm{H}^{-}\right.$s) up to $0.8 \mathrm{MeV}$. This RFQ employs a conventional beam dynamics design; that is, the intervane voltage $V$ and the average bore radius are maintained constant except for the injection section. The cell parameters were designed with KEKRFQ [25], and the number of the cells is 297. In order to use this RFQ for muon acceleration, the voltage $V$ should be normalized to the muon mass, and the input velocity $\beta$ should be the same as that of the $\mathrm{H}^{-}$, as shown in Table I.

This RFQ is longitudinally separated into two modules. Each module consists of upper and lower major vanes and left and right minor vanes made of oxygen-free copper. The vane tips and inside surfaces of the cavity were machined with formed cutters. The machining accuracy of the vane tips was within $15 \mu \mathrm{m}$. In the center port of the lower-right quadrant, a loop-type rf coupler was inserted. The rf power was set to $2.3 \mathrm{~kW}$ for the muon acceleration operation, and transmitted via $50-\Omega$ coaxial cables. The forward and reflected if powers were measured through a directional coupler attached to the rf coupler, and the rf power in the RFQ cavity was measured using a loop pickup monitor inserted into the cavity. Figure 2 shows the typical rf waveform of the RFQ. In this figure, the rf pulses input into (Forward) and reflected from (Reflection) the RFQ, the filled power in the RFQ, and the trigger for the waveform digitizer of the beam detector are indicated. The rf pulse width was $100 \mu \mathrm{s}$ and the repetition rate was $25 \mathrm{~Hz}$.

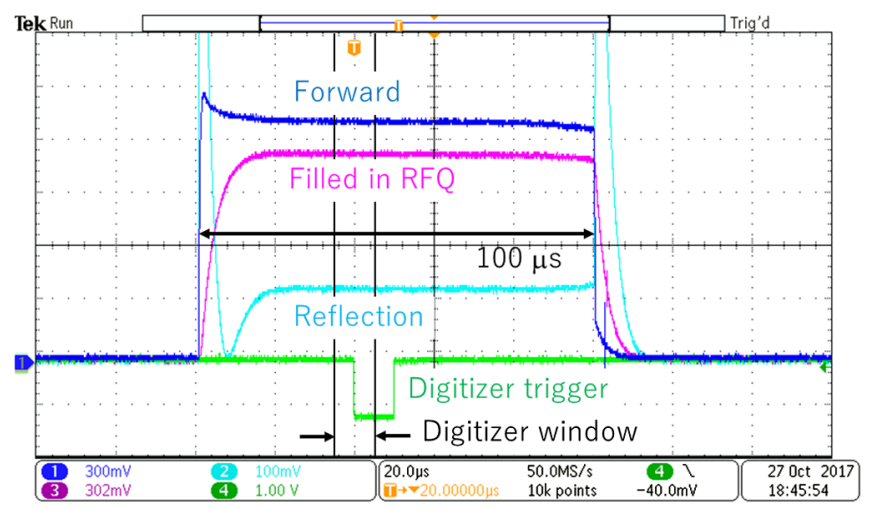

FIG. 2. Radio-frequency pulses input (Forward) into, reflected from, and filled in the RFQ. The digitizer window for the beam detector is also shown.

The extracted beam properties were measured using a beam diagnostics line. The beam was transferred using two quadrupole magnets (QM1 and QM2) with field gradients of 2.6 and $1.8 \mathrm{~T} / \mathrm{m}$, respectively. The charge and momentum of the particle can be selected with a bending magnet (BM). In our experiment, because the intensity of the $\mathrm{Mu}^{-}$ is very low, it is not feasible to find the correct field setting with the ordinary BM-current scanning method. Instead, we used $\mathrm{H}^{-}$'s generated by exposing the $\mathrm{Al}$ degrader surface to ultraviolet light. If the acceleration voltage of the Soa lens is set to $10 \mathrm{kV}$, the momentum of the $\mathrm{H}^{-}$'s is the same as that of the $89 \mathrm{keV} \mathrm{Mu}^{-}$'s. The field setting of the $\mathrm{BM}$ was verified using $10 \mathrm{keV} \mathrm{H}^{-}$prior to the muon acceleration experiment.

A microchannel plate (MCP, Hamamatsu photonics F9892-21 [26]) was located at the downstream end of the $45^{\circ}$ line. The fiducial area of the MCP detector corresponded to a circle of $42 \mathrm{~mm}$ diameter, and the aperture ratio of the micro channel was $60 \%$. The MCP waveform, in an interval of $10 \mu \mathrm{s}$ around each $25-\mathrm{Hz}$ beam pulse, was digitized with a $250 \mathrm{MS} / \mathrm{s}$ waveform digitizer. The $\pm 5 \mu \mathrm{s}$ from the leading edge of the digitizer trigger in Fig. 2 corresponds to the digitizing window. A pulse higher than the pedestal level of the waveform was regarded as a signal pulse. The leading edge of the signal pulse and the maximum height within the signal window of $40 \mathrm{~ns}$ were defined as the signal timing and pulse height, respectively.

\section{RESULT}

At the beginning of the muon acceleration experiment, the beam diagnostics system was verified using penetrating $\mu^{+}$'s. The energy spectrum of the penetrating $\mu^{+}$'s is continuous up to more than $90 \mathrm{keV}$, and $89 \pm 18 \mathrm{keV}$ $\mu^{+}$'s can be selected with the BM. Figure 3(a) is a scatter plot of pulse height vs time of flight (TOF) for the observed $\mu^{+}$with the MCP. The muon arrival time at the $\mathrm{Al}$ degrader was measured with a set of scintillating counters located at the side of the Al degrader. Figure 3(b) is a projection to the 


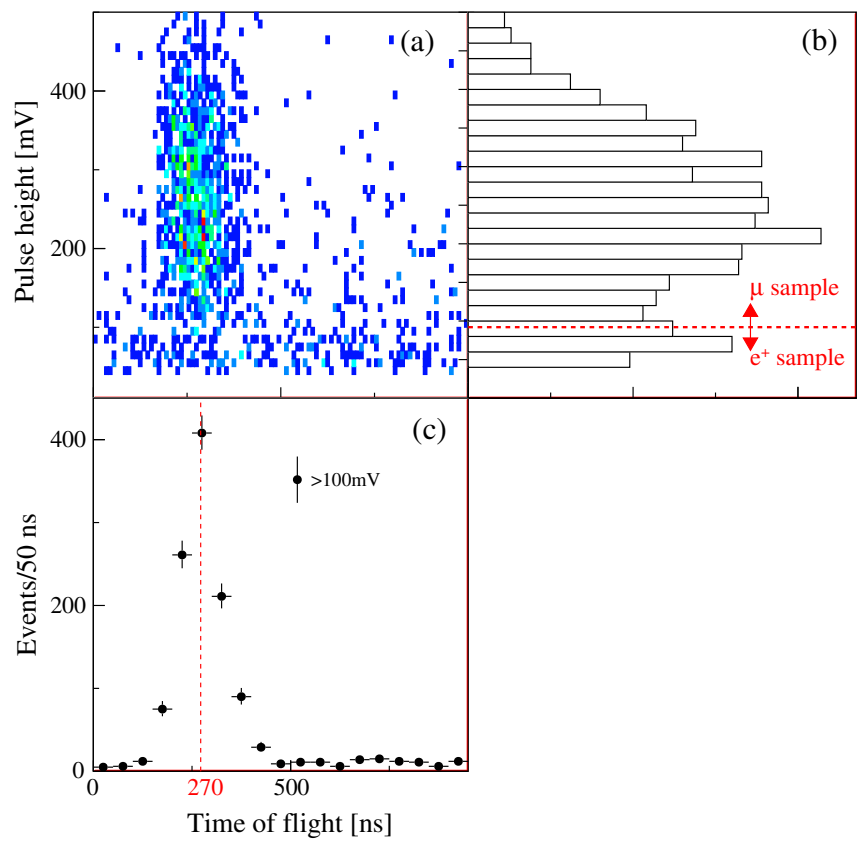

FIG. 3. Distribution of the MCP pulse height and the TOF of the penetrating $\mu^{+}$. (a) Scatter plot of the pulse height vs TOF. (b) Pulse height of the MCP signal. The events above $100 \mathrm{mV}$ were regarded as $\mu^{+}$. (c) TOF spectrum after the pulse-height cut was applied. The peak corresponds to the $\mu^{+}$'s injected into the RFQ with an energy of $89 \mathrm{keV}$.

pulse-height axis. The main background of the muon measurement is decay positrons from the $\mu^{+}$, but they penetrate the MCP, and thus are easily eliminated by applying a pulse-height cut. The threshold was determined from Figs. 3(a) and 3(b) as $100 \mathrm{mV}$. Figure 3(c) shows the TOF distribution after the pulse-height cut was applied. Because the distance between the $\mathrm{Al}$ degrader and the $\mathrm{MCP}$ is $3.4 \mathrm{~m}$, the TOF of the $89 \mathrm{keV} \mu^{+}$is $270 \mathrm{~ns}$. The observed TOF peak is consistent with this calculation. The rms timing width of $50 \mathrm{~ns}$ mainly comes from that of the primary muon beam at the $\mathrm{Al}$ degrader.

Finally, the polarities of the magnets were flipped to the negative-charge configuration. Figure 4 shows the TOF spectrum with and without the rf operation after the pulseheight cut was applied. With the rf operation, a clear peak was observed at $830 \pm 11 \mathrm{~ns}$. The error is the statistical error of the peak position of the Gaussian fitting. The number of cells of this 324-MHz RFQ is 297, and thus it takes $\frac{297}{2 \times 324 \times 10^{6}}=458 \mathrm{~ns}$ to fully accelerate the particles through the $324 \mathrm{MHz}$ RFQ. Therefore, the arrival time of the accelerated $\mathrm{Mu}^{-}$is later than that of the penetrated $\mu^{+}$.

The TOF spectrum was confirmed with a series of simulations. The simulation of the Soa lens was conducted using GEANT4 [27]. The three-dimensional electric field was calculated with OPERA3D [28] and implemented in the simulation. The transit time through the Soa lens was estimated with this simulation to be $307 \mathrm{~ns}$ and the

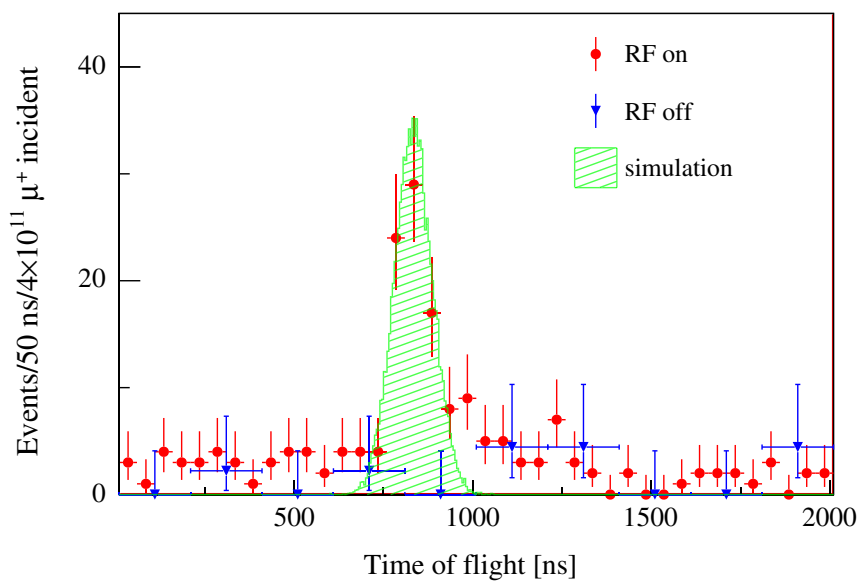

FIG. 4. TOF spectra of the negative-charge configuration with rf on and off. The clear peak of the rf on spectrum at $830 \mathrm{~ns}$ corresponds to the accelerated $\mathrm{Mu}^{-}$'s. The error bars are statistical. A simulated TOF spectrum of the accelerated $\mathrm{Mu}^{-}$'s is also plotted.

acceptance was estimated to be 4\%. PARMTEQM [29] was employed for the RFQ simulation, and the transmission was estimated to be $5 \%$. Almost all losses occurred at the RFQ entrance, because of much larger emittances than the acceptance of the RFQ. TRACE3D [30] and PARMILA [31] were utilized for the diagnostics line simulation. The transport efficiency to the $\mathrm{MCP}$ was evaluated to be $87 \%$. The length of the diagnostics line is $0.91 \mathrm{~m}$, and thus the transit time of the $89 \mathrm{keV} \mathrm{Mu}^{-}$is $72 \mathrm{~ns}$. The total flight time of the accelerated $\mathrm{Mu}^{-}$from the $\mathrm{Al}$ degrader to the MCP was calculated to be $t_{\text {tran }}=307+458+72=$ $837 \mathrm{~ns}$, which is consistent with the measurement. The hatched histogram in Fig. 4 represents the simulated TOF spectrum of the accelerated $\mathrm{Mu}^{-}$. The number of simulation events was normalized to $4 \times 10^{11}$ incident $\mu^{+}$'s. The muon survival rate was calculated to be $\exp \left(t_{\text {tran }} / \tau_{\mu}\right)=$ $81 \%$. The $46 \mathrm{~ns}$ rms width of the TOF spectrum is consistent with that from the timing distribution of the primary $\mu^{+}$at the $\mathrm{Al}$ degrader.

From these experimental results, it is concluded that the observed TOF peak is due to the $\mathrm{Mu}^{-}$'s accelerated by the RFQ to $89 \mathrm{keV}$. The event rate in the 780 to $980 \mathrm{~ns}$ TOF range was estimated to be $(5 \pm 1) \times 10^{-4} / \mathrm{s}$ by subtracting the decay-positron events estimated from the timing region outside the signal range.

\section{CONCLUSION}

In summary, muons have been accelerated by rf acceleration for the first time. Slow negative muonium atoms $\left(\mathrm{Mu}^{-}\right)$were generated through the electron capture process of the degraded $\mu^{+}$'s in the D2 area of J-PARC MUSE, and accelerated with the RFQ up to $89 \mathrm{keV}$.

The intensity of the accelerated $\mathrm{Mu}^{-}$in this experiment is limited by the very low conversion efficiency of $2.9 \mathrm{MeV}$ 
$\mu^{+}$to $\mathrm{Mu}^{-}$. With the construction of the new $\mathrm{H}$ line and assuming the design value of 1-MW beam power from the RCS, the intensity is expected to be $2 \times 10^{-2} / \mathrm{s}$. Structures to further accelerate the beam from the RFQ are now being developed, and can be demonstrated using this beam. There is also the possibility of significantly improving the conversion efficiency by cesiation as with $\mathrm{H}^{-}$ion sources [32]. Finally, the laser-dissociation ultraslow muon source is expected to be installed in this beam line to obtain a muon rate of $10^{6} / \mathrm{s}$, the design intensity for the $g-2 / \mathrm{EDM}$ experiment. The result presented in this paper is the first step toward making the low-emittance muon beam available as a powerful tool for application in material and life sciences and fundamental physics research.

\section{ACKNOWLEDGMENTS}

The authors would like to thank the J-PARC muon section staffs for their support in the conduct of the experiment at J-PARC MUSE. We also thank the J-PARC and KEKB linac groups for their support in assembling the accelerator components. We express our appreciation to the many manufacturing companies involved in this project, especially to Toshiba Co., who fabricated the RFQ. This work is supported by JSPS KAKENHI Grants No. JP25800164, No. JP15H03666, No. JP16H03987, No. JP15H05742, No. JP16K13810, No. JP16J07784, and No. JP18H03707. This work is also supported by the Korean National Research Foundation Grants No. NRF2015H1A2A1030275, No. NRF-2015K2A2A4000092, and No. NRF-2017R1A2B3007018; the Russian Foundation for Basic Research Grant No. RFBR 17-52-50064; and the Russian Science Foundation Grant No. RNF 17-12-01036. This experiment at the Materials and Life Science Experimental Facility of the J-PARC was performed under user programs (Proposal No. 2017A0263).

[1] M. A. Palmer, ICFA Beam Dynamics Newsletter, Vol. 55 (2011), p. 72.

[2] D. Neuffer, Principles and applications of Muon cooling, Part. Accel. 14, 75 (1983).

[3] http://slowmuon.kek.jp/MuonMicroscopy_e.html.

[4] G.W. Bennett et al., Final report of the E821 muon anomalous magnetic moment measurement at BNL, Phys. Rev. D 73, 072003 (2006).

[5] K. Hagiwara, R. Liao, A. D. Martin, D. Nomura, and T. Teubner, $(g-2) \mu$ and $\alpha\left(M^{2} Z\right)$ re-evaluated using new precise data, J. Phys. G 38, 085003 (2011).

[6] M. Davier, A. Hoecker, B. Malaescu, and Z. Zhang, Reevaluation of the hadronic contributions to the muon $g-2$ and to $\alpha\left(M_{Z}^{2}\right)$, Eur. Phys. J. C 71, 1 (2011); Erratum, Eur. Phys. J. C 72, 1874 (2012).

[7] M. Benayoun, P. David, L. DelBuono, and F. Jegerlehner, An update of the HLS estimate of the muon $g-2$, Eur. Phys. J. C 73, 2453 (2013).
[8] J-PARC E34 Conceptual Design Report, edited by T. Mibe, KEK, 2011.

[9] J. Grange et al., Muon $(g-2)$ Technical Design Report, arXiv:1501.06858.

[10] G. A. Beer et al., Enhancement of muonium emission rate from silica aerogel with a laser-ablated surface, Prog. Theor. Exp. Phys. (2014) 091C01.

[11] M. Otani, N. Kawamura, T. Mibe, M. Yoshida, K. Hasegawa, T. Ito, Y. Kondo, N. Hayashizaki, Y. Iwashita, Y. Iwata, R. Kitamura, and N. Saito, Development of a muon Linac for the G-2/EDM experiment at J-PARC, in Proceedings of LINAC2016, East Lansing, MI, 2016, (JACoW, East Lansing, MI, 2016) pp. 1037-1041.

[12] N. Kawamura, A. Toyoda, T. Mibe, N. Saito, M. Aoki, K. Shimomura, and Y. Miyake, $\mathrm{H}$ line; A beamline for fundamental physics in J-PARC, J. Phys. Soc. Jpn. Conf. Proc. 2, 010112 (2014).

[13] Y. Miyake, K. Shimomura, N. Kawamura, P. Strasser, S. Makimura, A. Koda, H. Fujimori, K. Nakahara, S. Takeshita, Y. Kobayashi, K. Nishiyama, W. Higemoto, T. U. Ito, K. Ninomiya, M. Kato, R. Kadono, N. Sato, and K. Nagamine, J-PARC muon facility, MUSE, J. Phys. Conf. Ser. 225, 012036 (2010).

[14] Y. Kondo, K. Hasegawa, M. Otani, T. Mibe, N. Saito, and R. Kitamura, Simulation study of muon acceleration using RFQ for a new muon g-2experiment at J-PARC, in Proceedings of IPAC2015, Richmond, VA, 2015 (JACoW, Richmond, VA, 2015), pp. 3801-3803.

[15] M. Otani, T. Mibe, M. Yoshida, K. Hasegawa, Y. Kondo, N. Hayashizaki, Y. Iwashita, Y. Iwata, R. Kitamura, and N. Saito, Interdigital H-mode drift-tube linac design with alternative phase focusing for muon linac, Phys. Rev. Accel. Beams 19, 040101 (2016).

[16] M. Otani, F. Naito, T. Mibe, M. Yoshida, K. Hasegawa, T. Ito, Y. Kondo, N. Hayashizaki, Y. Iwashita, Y. Iwata, R. Kitamura, and N. Saito, Development of muon Linac for th muon g-2/EDM experiment at J-PARC, in Proceedings of IPAC2016, Busan, Korea, 2016 (JACoW, Busan, Korea, 2016), pp. 1543-1546.

[17] Y. Kondo, K. Hasegawa, M. Otani, T. Mibe, M. Yoshida, and R. Kitamura, Beam dynamics design of the muon linac high-beta section, J. Phys. Conf. Ser. 874, 012054 (2017).

[18] H. Miyadera, A. J. Jason, and K. Nagamine, Design of muon accelerators for an advanced muon facility, in Proceedings of the 16th IEEE International Pulsed Power Conference, Albuquerque, NM (IEEE, Piscataway, NJ, 2007), pp. 3032-3034.

[19] Y. Kuang, K. P. Arnold, F. Chmely, M. Eckhause, V. W. Hughes, J. R. Kane, S. Kettell, D. H. Kim, K. Kumar, D. C. Lu, B. Matthias, B. Ni, H. Orth, G. Zu Putlitz, H. R. Schaefer, P. A. Souder, and K. Woodle, Formation of the negative muonium ion and charge-exchange processes for positive muons passing through thin metal foils, Phys. Rev. A 39, 6109 (1989).

[20] Y. Kondo, K. Hasegawa, and A. Ueno, Fabrication and low-power measurements of the J-PARC 50-mA RFQ prototype, in Proceedings of the 23rd International Linac Conference, LINAC-2006, Knoxville, TN, 2006 (JACoW, Knoxville, TN, 2006), pp. 749-751. 
[21] P. Strasser, K. Shimomura, A. Koda, N. Kawamura, H. Fujimori, S. Makimura, Y. Kobayashi, K. Nakahara, M. Kato, S. Takeshita, M. Hiraishi, M. Miyazaki, W. Higemoto, T. U. Ito, K. Ninomiya, K. Ishida, M. K. Kubo, R. Kadono, K. Nishiyama, and Y. Miyake, J-PARC decay muon channel construction status, J. Phys. Conf. Ser. 225, 012050 (2010).

[22] R. Kitamura, M. Otani, Y. Fukao, N. Kawamura, T. Mibe, Y. Miyake, K. Shimomura, Y. Kondo, K. Hasegawa, S. Bae, B. Kim, G. Razuvaev, H. Iinuma, K. Ishida, and N. Saito, First trial of the muon acceleration for J-PARC muon g-2/EDM experiment, J. Phys. Conf. Ser. 874, 012055 (2017).

[23] G4beamline, http://public.muonsinc.com/Projects/G4beamline .aspx.

[24] K. F. Canter, P. H. Lippel, W. S. Crane, and A. P. Mills Jr., Modified soa immersion lens positron gun, in Positron Studies of Solids, Surfaces and Atoms (World Scientific, Singapore, 1986), p. 199.
[25] A. Ueno and Y. Yamazaki, New beam-dynamics design procedure for RFQs, in Proceedings of the 1990 Linac Conference, Albuquerque, New Mexico, 1990 (JACoW, Albuquerque, New Mexico, 1990), pp. 329-331.

[26] Hamamatsu Photonics K. K., http://www.hamamatsu.com/.

[27] Geant4, http://geant4.cern.ch/.

[28] OPERA3D, Vector Fields Limited, Oxford, England, https://operafea.com/.

[29] K. R. Crandall et al., RFQ Design Codes, Report No. LAUR-96-1836, 1996.

[30] K. R. Crandall and D. P. Rusthoi, Trace 3-D Documentation, Report No. LA-UR-97-886, 1997.

[31] H. Takeda, Parmila, Report No. LA-UR-98-4478, 1998.

[32] V. Dudnikov, M. A.C. Cummings, R. P. Johnson, and A. D. Dudnikov, Cold muonium negative ion production, in Proceedings of IPAC2017, Copenhagen, Denmark, 2017 (JACoW, Copenhagen, Denmark, 2016), pp. 28982901. 